\title{
The role of cavity losses on non-adiabatic couplings and dynamics in polaritonic chemistry
}

\author{
Panayiota Antoniou, Figen Suchanek, James F. Varner, and Jonathan J. Foley \\ $\mathrm{IV}^{*}$ \\ Department of Chemistry, William Paterson University, 300 Pompton Road, Wayne, NJ, \\ $07470, U S A$ \\ E-mail: foleyj10@wpunj.edu
}

\begin{abstract}
We present a non-Hermitian formulation of the polaritonic structure of azobenzene strongly coupled to a photonic mode that explicitly accounts for the fleeting nature of the photonmolecule interaction. This formalism reveals that the polaritonic non-adiabatic couplings that facilitate cis-trans isomerization can be dramatically modified by photonic dissipation. We perform Fewest-Switches Surface Hopping dynamics on the surfaces that derive from our nonHermitian formalism and find that the polaritonic isomerization yields are strongly suppressed for moderate to large photon dissipation rates. These findings highlight the important role that the finite lifetime of photonic degrees of freedom play in polaritonic chemistry.
\end{abstract}

Molecular polaritons arise when the interaction between light and molecular transitions is large compared to the dissipative energy scales in the system. ${ }^{1-9}$ Strong coupling to photons can be realized by placing the molecular system in an optical cavity tuned to be resonant with a molecular transition, ${ }^{2,4,8}$ or in the vicinity of nanomaterials that support strong optical modes, including plasmon resonances ${ }^{1,10}$ or scattering-mediated absorption resonances. ${ }^{11}$ Ebbesen and co-workers demonstrated experimentally that the reactivity of polaritonic systems be dramatically different than that of the lone molecular system. ${ }^{2,5}$ Subsequently, a wealth of theoretical studies have suggested that strong coupling can reshape the energy landscape that dictates reactivity. $4,7,8,12-21$

Azobenzene has been studied as a prototypical molecule for photoisomerization that can be potentially used as a molecular photo-switch or energy storage material, among other applications. ${ }^{22,23}$ The excited-state dynamics of azobenzene accessible by visible-light excitation are guided by a quasi-harmonic potential along the isomerization coordinate $(R)$ with a minimum that approximately coincides with the transition-state value of $R$ on the ground-state surface. Hence, photochemical activation of cisazobenzene yields the trans- isomer with nearly equal likelihood as relaxation back to the cisisomer ${ }^{8,14}$ (see Figure 1).

Unlike traditional photochemistry where the interaction between light and molecules is perturbative, polaritonic chemistry occurs when light hybridizes with molecular degrees of freedom, causing the potential energy surfaces to inherit properties of the photonic and molecular systems. For example, polaritonic potential energy surfaces may develop new critical points, new intersections or avoided crossings, and different couplings between surfaces; these new features may suppress or enhance the reactivity compared to the lone molecular system. Azobenzene strongly coupled to a photon can illustrate all of these features depend- 
ing on the details of the photon and its confinement $^{4,7,8,17,19}$ (see Figure 1).

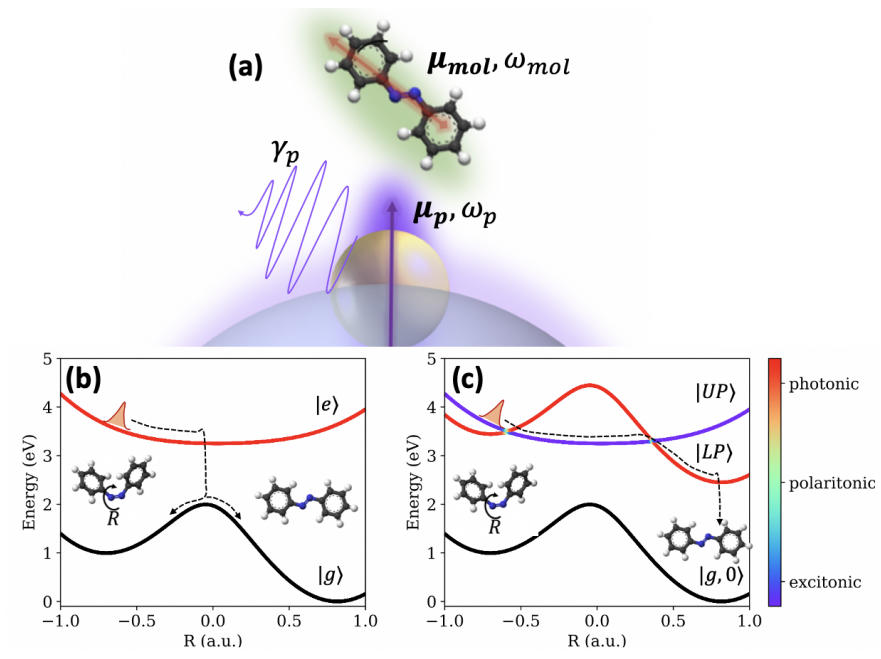

Figure 1: (a) Schematic of azobenzene coupled through its electronic transition dipole moment $\left(\mu_{m o l}\right)$ to the effective transition dipole moment of a localized photonic resonance in an optical nanostructure $\left(\mu_{p}\right)$. The frequency of the molecular transition $\left(\omega_{m o l}\right)$, the frequency of the photonic resonance $\left(\omega_{p}\right)$, and the dissipation rate of the photonic resonance $\left(\gamma_{p}\right)$ all impact the polaritonic structure. (b) The singlet ground- $(|g\rangle)$ and first excited-state $(|e\rangle)$ potential energy surfaces of azobenzene along the reaction coordinate $R$. (c) The composite singlet ground, zero-photon state $(|g, 0\rangle)$, lower polariton $(|L P\rangle)$ and upper polariton $(|U P\rangle)$ potential energy surfaces along $R$ that result when $\hbar \omega_{m o l}=\hbar \omega_{p}=2.45 \mathrm{eV}$ and $\hbar g=0.02 \mathrm{eV}$, where $\hbar g \propto \mu_{m o l} \cdot \mu_{p}$.

Polaritonic chemistry is an inherently multiscale problem that has motivated considerable theoretical and computational effort. In the case of electronic strong coupling, which is the focus of our work here, the photonic and molecular electronic degrees of freedom are often treated on the same quantum mechanical footing, while the nuclear degrees of freedom may be treated as slow or classical degrees of freedom. ${ }^{4,13,15,20}$ Drawing on this Cavity Born-Oppenheimer approximation, the nuclear dynamics in polaritonic chemistry have been simulated classically using Ehrenfest dynamics ${ }^{18}$ and Fewest-Switches Surface Hopping, ${ }^{17-20}$ and quantum mechanically using the framework of exact factorization, ${ }^{7}$ the partial linearized density matrix path-integral approach, ${ }^{8}$ and wavepacket propagation. ${ }^{21}$ The molecular electronic and photonic degrees of freedom have been treated using model and/or semi-empirical Hamiltonians, ${ }^{8,12,14,16,17}$ although there has been a recent surge in activity focused on merging $a b$ initio molecular electronic structure theory with cavity quantum electrodynamics ( $a b$ initio CQED) to provide an accurate and predictive model of polaritonic structure. ${ }^{13,24-29}$ The dissipative nature of the photonic degrees of freedom has been less extensively explored in terms of how they impact upon the polaritonic structure itself, and on the dynamics that occur on one or more polaritonic surfaces. Here we couple a non-Hermitian CQED Hamiltonian ${ }^{30}$ to a model Hamiltonian for the molecular electronic structure of azobenzene to simulate the polaritonic structure and dynamics with explicit inclusion of finite cavity lifetimes. We utilize fewest-switches surface hopping (FSSH) dynamics ${ }^{17,19,20,31-33}$ with potential energy surfaces and non-adiabatic couplings from our nonHermitian polaritonic structure theory to elucidate the impact of cavity lifetime on the isomerization dynamics under other cavity parameters previously found to facilitate facile cis-to-trans isomerization. ${ }^{8,17}$ A similar nonHermitian scheme to investigate cavity losses in polaritonic chemistry has been independently developed by Feist and co-workers. ${ }^{21}$ There, they employ full quantum simulations of the photorelaxation dynamics of uracil, and find conditions whereby dissipation and coupling strength conspire to optimize photoprotection. ${ }^{21}$ In the model azobenzene system we explore, we find that the isomerization yield is strongly suppressed for cavity dissipation rates greater than $\hbar \gamma \geq 5 \mathrm{meV}$. In particular, we find that the couplings between the polaritonic surfaces are quantitatively altered by the cavity dissipation rates, and that the dissipative features of the photon are inherited broadly by the polaritonic surfaces. The former effect impedes non-adiabatic transitions between the polaritonic surfaces that can facilitate isomerization, while the latter effect tends to favor relaxation to the ground-state surface, which also inhibits isomerization. Our approach sheds new 
light on the important role that cavity lifetime plays on polaritonic structure itself, and by extension, the dynamics that evolve on polaritonic surfaces.

We consider the prototypical system of azobenzene strongly coupled to a cavity that supports a photonic resonance. Following previous studies, ${ }^{8,17}$ we use a generalized Rabi Hamiltonian as follows:

$$
\hat{H}_{p l}=\hat{H}_{e l}+\hat{H}_{p}+\hat{H}_{e l, p}
$$

where $\hat{H}_{e l}$ denotes the electronic Hamiltonian:

$$
\hat{H}_{e l}=E_{g}(R) \hat{a}_{g}^{\dagger} \hat{a}_{g}+E_{e}(R) \hat{a}_{e}^{\dagger} \hat{a}_{e},
$$

where $E_{g}(R) \quad\left(E_{e}(R)\right)$ denotes the ground(excited-) state electronic energy at nuclear coordinate $R$, and $\hat{a}_{p}^{\dagger}\left(\hat{a}_{p}\right)$ are the creation (annihilation) operators for electronic state $p$. The nuclear coordinate $R$ here denotes the dihedral angle of the Ph-N-N-Ph system that defines the cis-trans isomerization (see Figure 1). The electronic states as a function of the nuclear coordinate are parameterized following the work of Mandal and $\mathrm{Huo}^{8}$ (see Supporting Information for more details).

The cavity resonances have finite lifetimes, which we model here by attributing a complex frequency to the photonic degrees of freedom. We write the photonic Hamiltonian as

$$
\hat{H}_{p}=\hbar\left(\omega-i \frac{\gamma}{2}\right) \hat{b}^{\dagger} \hat{b}
$$

where $\hat{b}^{\dagger}(\hat{b})$ denote the raising (lowering) operator for the photon state with complex frequency $\tilde{\omega}=\omega-i \frac{\gamma}{2}$, where $\gamma$ arises from the finite-lifetime of the photonic resonance. We parameterize the photonic Hamiltonian to have $\hbar \omega=2.45 \mathrm{eV}$ and $\hbar \gamma$ to have values in the range 0.02 to $100 \mathrm{meV}$. Such a non-Hermitian form of this photonic Hamiltonian was recently considered by Cortes, Otten, and Gray ${ }^{30}$ and captures important dissipative effects if the photonic degrees of freedom. Finally, the interaction Hamiltonian has the form ${ }^{8,17,30}$

$$
\hat{H}_{e l, p}=\hbar g\left(\hat{b}^{\dagger}+\hat{b}\right)\left(\hat{a}_{e}^{\dagger} \hat{a}_{g}+\hat{a}_{g}^{\dagger} \hat{a}_{e}\right) ;
$$

in this work, we neglect the dipole self-energy term in the polaritonic Hamiltonian, which has recently been found to play a key role in the polaritonic structure and dynamics of systems with multiple photonic modes ${ }^{34}$ and/or systems in the ultra-strong coupling regime. ${ }^{35}$ Here we consider a 1-photon 1-molecule system in which the coupling term has the value $\hbar g=0.02 \mathrm{eV}$ (i.e. not in the ultra-strong coupling regime); in recent work by Mandal and Huo, this coupling along with a photon energy of $2.45 \mathrm{eV}$ was shown to be particularly facile for polaritonic isomerization. ${ }^{8}$

We can express the Hamiltonian matrices in a local basis that includes states where energy is localized as a photon in the cavity mode, in the molecular system as an exciton, in both, or in neither. In other words, this system permits the following four composite basis states $\left|\phi_{i}\right\rangle \in$ $\{|g, 0\rangle,|g, 1\rangle,|e, 0\rangle,|e, 1\rangle\}$, where the composite state $\left|\phi_{1}\right\rangle=|g, 0\rangle$ denotes the molecule is in its ground state and no photon in the cavity.

In this basis, the Hamiltonian matrices for our 1-photon 1-molecule system can be written as

$$
\mathbf{H}_{e l}=\left(\begin{array}{cccc}
E_{g}(R) & 0 & 0 & 0 \\
0 & E_{g}(R) & 0 & \\
0 & 0 & E_{e}(R) & 0 \\
0 & 0 & 0 & E_{e}(R)
\end{array}\right)
$$

for the molecular electronic part,

$$
\mathbf{H}_{p}=\left(\begin{array}{cccc}
0 & 0 & 0 & 0 \\
0 & \hbar \tilde{\omega} & 0 & 0 \\
0 & 0 & 0 & 0 \\
0 & 0 & 0 & \hbar \tilde{\omega}
\end{array}\right)
$$

for the photonic part, and

$$
\mathbf{H}_{e l, p}=\left(\begin{array}{cccc}
0 & 0 & 0 & \hbar g \\
0 & 0 & \hbar g & 0 \\
0 & \hbar g & 0 & 0 \\
\hbar g & 0 & 0 & 0
\end{array}\right)
$$

for the interaction between the molecule and the cavity mode. The total Hamiltonian $\mathbf{H}_{\text {Tot }}$ in the local basis is the sum of all three contributions.

The new polaritonic potential energy surface can be identified by building $\mathbf{H}_{\text {Tot }}$ at different 
values of $R$, diagonalizing the matrix, and identifying the eigenvalues $\epsilon_{\alpha}(R)$; similarly, the energy eigenstates in the polariton basis, $\left|\Phi_{\alpha}(R)\right\rangle$, will be the eigenvectors of this matrix. We therefore refer to the basis that diagonalizes $\mathbf{H}_{T o t}$ as the polariton basis, and denote the Hamiltonian in this basis as $\mathbf{H}_{P l}$. At geometries where $E_{g}(R)+\hbar \tilde{\omega} \approx E_{e}(R)$, we get strong mixing of the $|g, 1\rangle$ and $|e, 0\rangle$ states. That is, $\left|\Phi_{2}\right\rangle$ and $\left|\Phi_{3}\right\rangle$ will be linear combinations of the form

$$
\left|\Phi_{\alpha}(R)\right\rangle=\sum_{i} c_{i}^{\alpha}(R)\left|\phi_{i}\right\rangle,
$$

that will typically have strong contributions coming from basis states $\left|\phi_{i}\right\rangle \in\{|g, 1\rangle,|e, 0\rangle\}$ at values of $R$ where the photon is resonant with the excitonic transition energy. We also refer to states $\left|\Phi_{2}\right\rangle$ and $\left|\Phi_{3}\right\rangle$ as $|L P\rangle$ and $|U P\rangle$, respectively, to denote their polaritonic character (see Figure 1). These polariton states will have associated potential energy surfaces defined by $\epsilon_{2}(R)$ and $\epsilon_{3}(R)$ that may differ qualitatively from the uncoupled surfaces, potentially imparting different reactivity to the system. Similar to previous studies, ${ }^{8,14}$ we can also elucidate the nature of the polaritonic surfaces as a function of geometry; i.e. some regions of the surface will take on a strong photonic character (when $\left|c_{2}^{\alpha}(R)\right|^{2} \approx 1$ ), some will take on a strong excitonic character $\left(\left|c_{3}^{\alpha}(R)\right|^{2} \approx 1\right)$, and others a mixed or polaritonic character $\left(\left|c_{2}^{\alpha}(R)\right|^{2} \approx\right.$ $\left.\left|c_{3}^{\alpha}(R)\right|^{2} \approx 0.5\right)$. That said, for all geometries, $\left|\Phi_{1}(R)\right\rangle \approx|g, 0\rangle$ and $\left|\Phi_{4}(R)\right\rangle \approx|e, 1\rangle$ with eigenvalues $\epsilon_{1}(R) \approx E_{g}(R)$ and $\epsilon_{4}(R) \approx$ $E_{e}(R)+\hbar \omega$.

We will see that the finite lifetimes of the photonic degrees of freedom are manifest in two key ways that impact polaritonic dynamics: (1) by modulating the relaxation back to the groundstate $\left|\Phi_{1}(R)\right\rangle$, which has a potential energy surface characterized by high barriers to isomerization compared to the polaritonic surfaces, and (2) by modulating the nature of the polaritonic surfaces themselves, including their degree of non-adiabatic coupling. The lifetime of the cavity modes in such polaritonic systems therefore is an interesting target of investigation since it can be controlled to large extent by the design of the cavity.

\section{Polaritonic Dynamics}

We track the nuclear motion along a single reactive coordinate $R$ using fewest-switches surface hopping (FSSH) dynamics. ${ }^{17,19,20,31-33}$ In this approach, the nuclear degree of freedom evolves according to classical dynamics, and the electronic/photonic degrees of freedom evolve according to quantum mechanics. Langevin dynamics are used for the nuclear degree of freedom to phenomenologically model vibrational relaxation. ${ }^{36-39}$ In our Surface Hopping scheme, the nuclear degree of freedom experiences two forces: a force from an active polaritonic surface, $F_{\lambda}(R)$ where $\lambda$ denotes the active surface, and a random force, $F_{r}(t)$. The force from the active polaritonic surface is given by

$$
F_{\lambda}(R)=-\left\langle\Phi_{\lambda}(R)\left|\mathbf{H}_{P l}^{\prime}\right| \Phi_{\lambda}(R)\right\rangle
$$

where $\left|\Phi_{\lambda}\right\rangle$ is the normalized active polaritonic eigenstate and $\mathbf{H}_{P l}^{\prime}=\frac{\partial}{\partial R} \mathbf{H}_{P l}$ is the derivative of the Hamiltonian in the polariton basis with respect to the nuclear coordinate $R$. This polaritonic force is real within the HellmanFeynman approximation in Equation 9. The random force is defined as

$$
F_{r}(t)=\sqrt{\frac{2 T \Gamma M}{d t}} Z(t),
$$

where $T$ is the temperature, $\Gamma$ is a friction term, $M$ is the effective mass of the nuclear degree of freedom, $d t$ is the simulation time-step, and $Z(t)$ is a Gaussian random variable with mean 0 and standard deviation of 1 . Values of all parameters are given in the Supporting Information.

Taking these two forces, the acceleration along the nuclear coordinate is determined from the Langevin equation of motion,

$$
\ddot{R}(t)=\frac{F_{\lambda}(R)+F_{r}(t)}{M}-\Gamma \dot{R}(t),
$$

where $\Gamma$ leads to a frictional force proportional to the nuclear velocity, $\dot{R}$.

The polaritonic degrees of freedom are represented quantum mechanically by a wavefunction that can be written as a superposition of 
polariton eigenstates,

$$
|\Psi(R, t)\rangle=\sum_{\alpha} C_{\alpha}(t)\left|\Phi_{\alpha}(R)\right\rangle .
$$

The time-dependence of these coefficients obeys the time-dependent Schrödinger equation,

$$
\dot{\mathbf{C}}(t)=-\frac{i}{\hbar} \mathbf{H}_{P l} \cdot \mathbf{C}(t)-\dot{R} \mathbf{d} \cdot \mathbf{C}(t),
$$

where $\mathbf{d}$ denotes the derivative coupling matrix, ${ }^{32}$ and $\mathbf{H}_{P l}$ is the non-Hermitian polariton Hamiltonian matrix; the dependence on the nuclear coordinate for both of these matrices is implied. The elements of the derivative coupling matrix are defined as

$$
d_{\alpha, \beta}(R)=-\frac{\left\langle\Phi_{\alpha}\left|\mathbf{H}_{P l}^{\prime}\right| \Phi_{\beta}\right\rangle}{E_{\beta}-E_{\alpha}} .
$$

Although the polaritonic wavefunction is typically in a superposition of multiple polaritonic eigenfunctions, in the FSSH scheme, the nuclei feel a force from a single polaritonic surface at a time, but can transition or "hop" between surfaces with a probability that is updated at each time-step. We compute the probability for hopping from surface $\alpha$ to $\beta$ to be given by

$$
P_{\alpha, \beta}(t)=\max \left(\frac{\dot{\rho}_{\beta}(t) d t}{\rho_{\alpha}(t)}, 0\right) .
$$

where $\dot{\rho}_{\beta}(t)$ is the time-derivative of the population of the polaritonic state $\Phi_{\beta}$ at time $t$ and $\rho_{\alpha}(t)$ is the population of polaritonic state $\Phi_{\alpha}$ at time $t$, defined as $\rho_{\alpha}(t)=C_{\alpha}^{*}(t) C_{\alpha}(t)$. At each time-step, as uniform random number $\xi \in[0,1]$ is chosen, and a surface hop to state $\beta<\alpha$ is performed when

$$
\sum_{i=1}^{\beta-1} P_{\alpha, i}<\xi \leq \sum_{i=1}^{\beta} P_{\alpha, i} .
$$

An average over a large number of such FSSH trajectories can provide a reasonable approximation for the true quantum dynamics of the system. ${ }^{19,20,31-33}$

We compute the polaritonic potential energy surfaces along the reaction coordinate $R$ for a variety of different values of $\hbar \gamma$ spanning low- loss $(<=1 \mathrm{meV})$ to high loss $(>=50 \mathrm{meV})$, where the high-loss cases are typical of plasmonic cavities and the low-loss cases could be realized in dielectric micro-resonators. ${ }^{10,11,40-42}$ In all calculations, $\hbar \omega=2.45 \mathrm{eV}$ and $\hbar g=0.02$ $\mathrm{eV}$. In Figure 2, the polaritonic surfaces are shown when $\hbar \gamma=1 \mathrm{meV}$ (panel (a) and (c), and $\hbar \gamma=100 \mathrm{meV}$ (panel (b) and (d)). Two qualitative features of the surfaces are altered by the magnitude of $\gamma$ : the avoided crossing between the surfaces under low-loss conditions becomes an intersection with high-loss, and the polaritonic character near the crossing feature is strongly diminished in the high-loss case (see Figure 2). Inspection of the derivative cou-
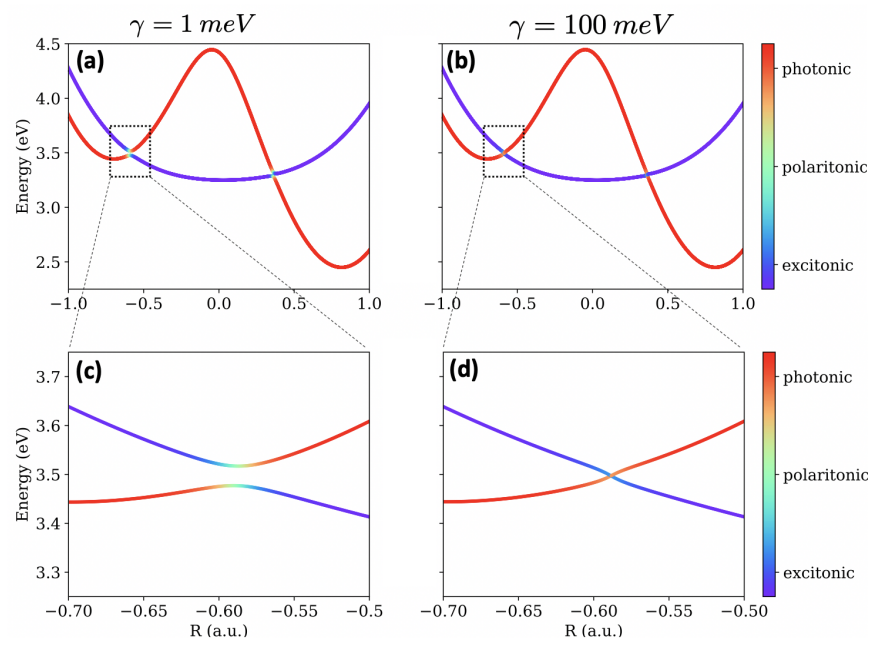

Figure 2: Polaritonic potential energy surfaces when $\hbar \gamma=1 \mathrm{meV}$ (a) and (c), and when $\hbar \gamma=100 \mathrm{meV}$ (b) and (d)

pling between the upper-polariton and lowerpolariton surfaces (i.e. $d_{3,2}$ or $d_{U P, L P}$ reveals that the magnitude of the derivative coupling in the vicinity of the crossing features is also strongly suppressed under large loss conditions (see Figure S1). The derivative coupling terms are directly responsible for transitions between surfaces, so one interesting consequence of coupling the molecular system to a high-loss cavity is the suppression of transitions between different polaritonic surfaces.

In the case of of the azobenzene under cavity coupling conditions used in this work, the transition between the $|U P\rangle$ and $|L P\rangle$ surfaces provides an energetically downhill reaction path that can facilitate nearly $100 \%$ yield for the 
cis-to-trans isomerization ${ }^{8,14,17,19}$ (see Figure S2 (a)). When these transitions are suppressed, the nuclear dynamics will tend to remain on the $|U P\rangle$ surface trapped in the cis configuration (see Figure S2 (b)). We therefore hypothesize that increasing cavity losses should inhibit cis-to-trans isomerization in the polaritonic azobenzene system, departing from prior results that included losses a posteriori. ${ }^{17,19}$

To explore the extent to which cavity losses impact the cis-to-trans isomerization yield, we utilize FSSH dynamics ${ }^{19,20,31-33}$ to simulate the polaritonic isomerization across a range of cavity loss values between 0.02 and $10 \mathrm{meV}$. For each cavity loss value, we run 500 FSSH trajectories starting in the cis configuration on the $|U P\rangle$ surface. The random distribution of initial positions and velocities for these trajectories are discussed in the Supporting Information. We find that the cis-to-trans isomerization rate approaches $100 \%$ as the cavity loss rate approaches 0 , but quickly declines with increasing loss rate (see Figure 3 ). In fact, we find the iso-

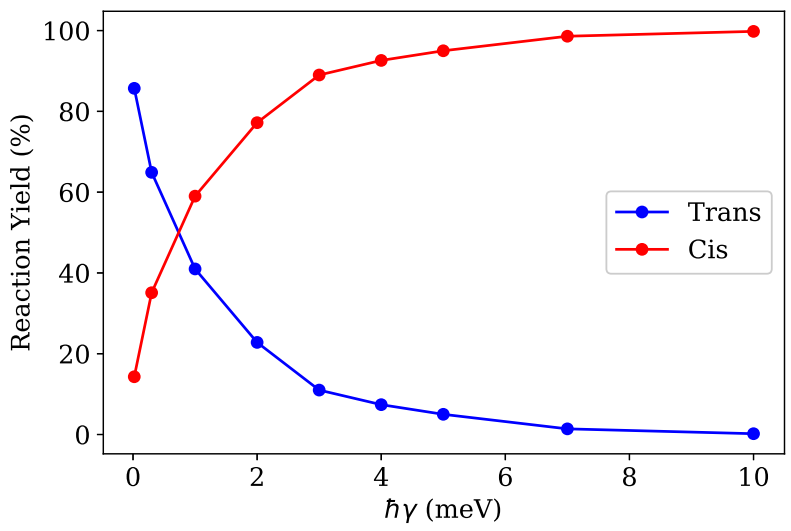

Figure 3: The isomerization yield for surface hopping trajectories initialized in the cis- configuration on the $|U P\rangle$ surface, showing a monotonic decrease in isomerization yield to the trans-isomer with increasing cavity dissipation rate.

merization yield is approximately $50 \%$ with a loss rate of $\hbar \gamma=1 \mathrm{meV}$, and that that the isomerization is almost completely suppressed for loss rates $\hbar \gamma \geq 5 \mathrm{meV}$. This result seems surprising in light of the fact that the absolute magnitude of the derivative coupling remains large for these modest loss rates, but investigation of the imaginary part of the derivative coupling between the $|U P\rangle$ and $|L P\rangle$ surfaces, and the imaginary part of the $|U P\rangle$ surface itself, provides further insights. We illustrate the
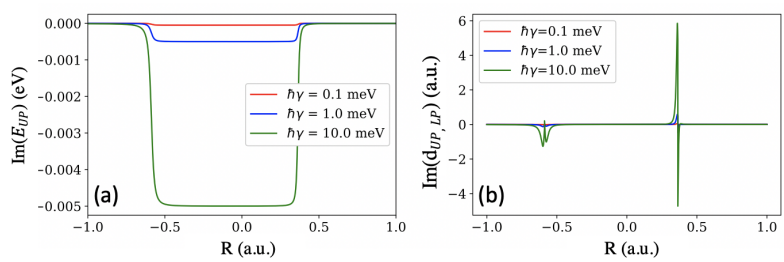

Figure 4: (a) Imaginary part of the upper-polariton potential energy surface $\left(\epsilon_{U P}\right)$ as a function of reaction coordinate $R$ for different values of the dissipation rate, showing that the cavity dissipation is inherited broadly by the upper-polariton surface. (b) Imaginary part of the derivative coupling matrix element between the upper- and lower-polariton surface along the reaction coordinate for different values of the dissipation rate. The increasing imaginary component of the derivative coupling dampens the nonadiabatic transitions between the polaritonic surfaces that can facilitate isomerization.

imaginary part of the $|U P\rangle$ surface around the values of the reaction coordinate that correspond to the cis isomer where the FSSH trajectories are initiated in Figure 4(a), and the imaginary part of the derivative coupling between the upper- and lower-polariton surfaces across all relevant values of $R$ in Figure 4(b). We find that the upper-polariton surface inherits the dissipative features of the photonic degrees of freedom across a fairly broad range of $R$ values about the cis configuration, as indicated by imaginary components of the PES on the oder of $\hbar \gamma / 2$ in each case. The regions of the $|U P\rangle$ surface that are more dissipative have more photonic character, whereas the regions that are less dissipative have more of the character of the molecular excited state (i.e. greater excitonic character, see the color scheme in Figure 2 and S2). The dissipation rate $\hbar \gamma=10$ meV shown in the green curve in Figure 4(a) will tend to cause relaxation to the ground-state (via surface hopping events from the $|U P\rangle \rightarrow$ $|g, 0\rangle)$ on the sub-picosecond timescale, whereas the isomerization itself occurs on a timescale of several picoseconds. Therefore, the dissipation timescale becomes faster than the isomerization timescale when the cavity loss rate is 
$\hbar \gamma 10 \mathrm{meV}$. This result is not novel in itself; for example, Fregoni et al. ${ }^{17}$ discussed regions of the upper-polariton surface being subject to large dissipation when cavity losses become large. In this study, the onset of dissipation in the $|U P\rangle$ surface is accompanied by the onset of large derivative couplings between the $|U P\rangle$ and $|L P\rangle$ surfaces that tend to drive transitions to the $|L P\rangle$ surface, which takes on more excitonic character precisely when the $|U P\rangle$ surface takes on more photonic character (see the color scheme in Figure 2 and Figure S2). Therefore, their study reported that transitions to the $|L P\rangle$ surface at around $R \approx-0.6$ a.u. tend to protect the dynamics from relaxation to the $|g, 0\rangle$ surface, and tend to allow downhill dynamics to the trans configuration. ${ }^{17}$ Where our non-Hermitian formulation of the polaritonic structure offers a new insight is in the computation of the derivative couplings between the $|U P\rangle$ and $|L P\rangle$ surfaces. As discussed previously, the absolute magnitude of the derivative couplings can be strongly altered for large cavity dissipation rates, but this does not explain the suppression of isomerization at the relatively modest dissipation rates of $\hbar \gamma \approx 10$ meV that we observe. We see that the derivative couplings pick up an imaginary component that grows in magnitude with $\hbar \gamma$; these imaginary components will tend to dampen the impact of the derivative coupling on the polaritonic wavefunction, thereby suppressing transitions between the $|U P\rangle$ and $|L P\rangle$ surfaces in the vicinity where the imaginary part becomes large. Incidentally, the imaginary part of $d_{U P, L P}$ becomes large in the vicinity of the avoid crossings where transitions are most likely under no- or low-loss conditions. Consequently, we find the trajectories that are initiated on the $|U P\rangle$ surface are unable to transition to the $|L P\rangle$ surface for moderate- to high loss rates, and this circumvents the dynamics that avoid relaxation to the $|g, 0\rangle$ state and ultimately facilitate cis-to-trans isomerization in the low- to no-loss cases.

\section{Conclusion}

We have developed a non-Hermitian formulation of polaritonic structure for the paradigmatic system of azobenzene coupled to a cavity that explicitly accounts for cavity losses. Our approach demonstrates that several features of the polaritonic structure are altered by the dissipative features of the cavity, and that these modifications can have profound impacts on the isomerization dynamics in this system. In particular, we find that cavity-enhanced isomerization reported in prior works is maintained only when photonic dissipation rates are $\leq 1$ meV, though this does not necessarily preclude favorable balances between coupling and dissipation that could allow polaritonic chemical control with larger photonic dissipation rates. These results suggest that explicit inclusion of cavity losses are critical for simulating polaritonic chemistry, and also points to the importance of loss engineering in optical cavities for polaritonic chemistry applications.

Acknowledgement This work was performed, in part, utilizing resources at the Center for Nanoscale Materials, a US Department of Energy,Office of Science, Office of Basic Energy Sciences User Facility (contract no. DE-AC02-06CH11357). High-performance computing resources of the MERCURY Consortium (http://www.mercuryconsortium.org) were used. JJF and FS acknowledge the Research Corporation for Scientific Advancement Cottrell Scholar Award for support. JJF, JFV and PA acknowledge the donors of the American Chemical Society Petroleum Research Fund for partial support of this work. JJF acknowledges the ART program at William Paterson University for partial support of this work.

\section{References}

(1) Bellessa, J.; Bonnand, C.; Plenet, J. C.; Mugnier, J. Strong coupling between surface plasmons and excitons in an organic semiconductor. Phys. Rev. Lett 2004, 93, 036404 . 
(2) Hutchison, J. A.; Schwartz, T.; Genet, C.; Devaux, E.; Ebbesen, T. W. Modifying Chemical Landscapes by Coupling to Vacuum Fields. Angew Chem Int Ed. 2012, 51, 1592-1596.

(3) Schwartz, T.; Hutchison., J.; Leonard, J.; Genet, C.; Haacke, S.; Ebbesen, T. Polariton Dynamics under Strong LightMolecule Coupling. Chem. Phys. Chem 2013, 14, 125-131.

(4) Galego, J.; Garcia-Vidal, F. J.; Feist, J. Cavity-induced modifications of molecular structure in the strong-coupling regime. Phys. Rev. X 2015, 5, 041022.

(5) Ebbesen, T. W. Hybrid light-matter states in a molecular and material science perspective. Acc. Chem. Res. 2016, 49, 24032412.

(6) Thomas, R.; Thomas, A.; Pullanchery, S.; Joseph, L.; Somasundaran, S. M.; Swathi, R. S.; Gray, S. K.; Thomas, K. G. Plexitons: The role of oscillator strengths and spectral widths in determining strong coupling. ACS Nano 12, 402-415.

(7) Hoffmann, N. M.; Appel, H.; Rubio, A.; Maitra, N. T. Light-matter interactions via the exact factorization approach. Eur. Phys. J. B 2018, 91, 180.

(8) Mandal, A.; Huo, P. Investigating new reactivities enabled by polariton photochemistry. J. Phys. Chem. Lett. 2019, 10, 5519-5529.

(9) Du, M.; Ribeiro, R. F.; Yuen-Zhou, J. Remote control of chemistry in optical devices. Chem. Cell. Press 2019, 5, 11671181.

(10) Thomas, R.; Thomas, A.; Pullanchery, S.; Joseph, L.; Somasundaran, S. M.; Swathi, R. S.; Gray, S. K.; Thomas, K. G. Plexcitons: The Role of Oscillator Strengths and Spectral Widths in Determining Strong Coupling. ACS Nano 2018, 0, null.
(11) Eldabagh, N.; Micek, M.; III, A. E. D. Resonance energy transfer mediated by metal-dielectric composite nanostructures. J. Phys. Chem. C 2018, 122, 18256-18265.

(12) Herrera, F.; Spano, F. C. Cavitycontrolled chemistry in molecular ensembles. Phys. Rev. Lett. 2016, 116, 238301.

(13) Flick, J.; Ruggenthaler, M.; Appel, H.; Rubio, A. Atoms and molecules in cavities, from weak to strong coupling in quantum-electrodynamics (QED) chemistry. Proc. Natl. Acad. Sci. USA 2017, 114, 3026-3034.

(14) Galego, J.; Garcia-Vidal, F. J.; Feist, J. Many-molecule reaction triggered by a single photon in polaritonic chemistry. Phys. Rev. Lett. 2017, 119, 136001.

(15) Flick, J.; Appel, H.; Ruggenthaler, M.; Rubio, A. Cavity Born-Oppenheimer approximation for correlated electron-nuclear-photon systems. Journal of Chemical Theory and Computation 2017, 13, 1616-1625.

(16) Martinez-Martinez, L. A.; Ribeiro, R. F.; Campos-Gonzalez-Angulo, J.; YuenZhou, J. Can ultrastrong coupling change ground-state chemical reactions? ACS Photonics 2018, 5, 167-176.

(17) Fregoni, J.; Granucci, G.; Coccia, E.; Persico, M.; Corni, S. Manipulating azobenzene photoisomerization through strong light-molecule coupling. Nat. Commun. 2018, 9, 4688.

(18) Zhang, Y.; Nelson, T.; Tretiak, S. Nonadiabatic molecular dynamics of molecules in the presence of strong light-matter interactions. The Journal of Chemical Physics 2019, 151, 154109.

(19) Fregoni, J.; Granucci, G.; Persico, M.; Corni, S. Strong coupling with light enhances the photoisomerization quantum yield of azobenzene. Chem 2020, 6, 250265. 
(20) Fregoni, J.; Corni, S.; Persico, M.; Granucci, G. Photochemistry in the strong coupling regime: A trajectory surface hopping scheme. J. Comput. Chem. 2020, 41, 2033-2044.

(21) Felicetti, S.; Fregoni, J.; Schnappinger, T.; Reiter, S.; de Vivie-Riedle, R.; Feist, J. Photoprotecting uracil by coupling with lossy nanocavities. arXiv 2020, $2007.07551 \mathrm{v} 1$.

(22) Bandara, H. M. D.; Burdette, S. C. Photoisomerization in Different Classes of Azobenzene. Chem. Soc. Rev 2012, 41, 1809-1825.

(23) Baroncini, M.; Groppi, J.; Corra, S.; Silvi, S.; A.Credi, Light-Responsive (Supra)Molecular Architectures: Recent Advances. Adv. Opt. Mat 2019, 7, 1900392.

(24) Tokatly, I. V. Time-dependent density functional theory for many-electron systems interacting with cavity photons. Phys. Rev. Lett. 2013, 110, 23301.

(25) Flick, J.; Narang, P. Cavity-correlated electron-nuclear dynamics from first principles. Phys. Rev. Lett. 2018, 121, 113002.

(26) Buchholz, F.; Theophilou, I.; Nielsen, S. E. B.; Ruggenthaler, M.; Rubio, A. Reduced density-matrix approach to strong matter-photon interaction. ACS Photonics 2019, 6, 2694-2711.

(27) Mordovina, U.; Bungey, C.; Appel, K.; Knowles, P. J.; Rubio, A.; Manby, F. R. Polaritonic coupled-cluster theory. Phys. Rev. Research 2020, 2, 023262.

(28) Rivera, N.; Flick, J.; Narang, P. Variational theory of nonrelativistic quantum electrodynamics. Phys. Rev. Lett. 2019, 122, 193603.

(29) Wang, D. S.; Neuman, T.; Flick, J.; Narang, P. Weak-to-strong light-matter coupling and dissipative dynamics from first principles. arXiv 2020, 2002, 10461v1.
(30) Cortes, C. L.; Otten, M.; Gray, S. K. Non-Hermitian approach for quantum plasmonics. J. Chem. Phys. 2020, 152, 084105.

(31) Tully, J. C. Molecular dynamics with electronic transitions. J. Chem. Phys. 1990, 93, 1061 .

(32) Subotnik, J. E.; Jain, A.; Landry, B.; Petit, A.; Ouyang, W.; Bellonzi, N. Understanding the surface hopping view of electronic transitions and decoherence. Annu. Rev. Phys. Chem. 2016, 67, 387-417.

(33) Tempelaar, R.; van der Vegte, C. P.; Knoester, J.; Jansena, T. L. C. Surface hopping modeling of two-dimensional spectra. J. Chem. Phys. 2013, 138, 164106.

(34) Hoffman, N. M.; Lacombe, L.; Rubio, A.; Maitra, N. T. Effect of many modes on self-polaritzation and photochemical suppression in cavities. arXiv 2020, 2001.07330.

(35) Mandal, A.; Krauss, T. D.; Huo, P. Polariton-mediated electron transfer via cavity quantum electrodynamics. J. Phys. Chem. B 2020,

(36) Oxtoby, D. W. Vibrational population relaxation in liquids. Adv. Chem. Phys. 1981, 47, 487.

(37) Kenkre, V. M.; Tokmakoff, A.; Fayer, M. D. Theory of vibrational relaxation of polyatomic molecules in liquids. J. Chem. Phys. 1994, 101, 10618.

(38) Skinner, J. L. Semiclassical approximations to golden rule rate constants. $J$. Chem. Phys. 1997, 10\%, 8717.

(39) Egorov, S. A.; Rabani, E.; Berne, B. J. Nonradiative relaxation processes in condensed phases: Quantum versus classical baths. J. Chem. Phys. 1999, 110, 5238. 
(40) Pan, F.; Smith, K. C.; Nguyen, H. L.; Knapper, K. A.; Masiello, D. J.; Goldsmith, R. H. Elucidating energy pathways through simultaneous measurement of absorption and transmission in a coupled plasmonic-photonic cavity. Nano Lett. 2020, 20, 50-58.

(41) Smith, H. T.; Karam, T. E.; Haber, L. H.; Lopata, K. Capturing Plasmon-Molecule Dynamics in Dye Monolayers on Metal Nanoparticles Using Classical Electrodynamics with Quantum Embedding. The Journal of Physical Chemistry C 2017, 121, 16932-16942.

(42) Nascimento, D. R.; DePrince III, A. E. Modeling molecule-plasmon interactions using quantized radiation fields within time-dependent electronic structure theory. J. Chem. Phys. 2015, 143, 214104. 
Graphical TOC Entry

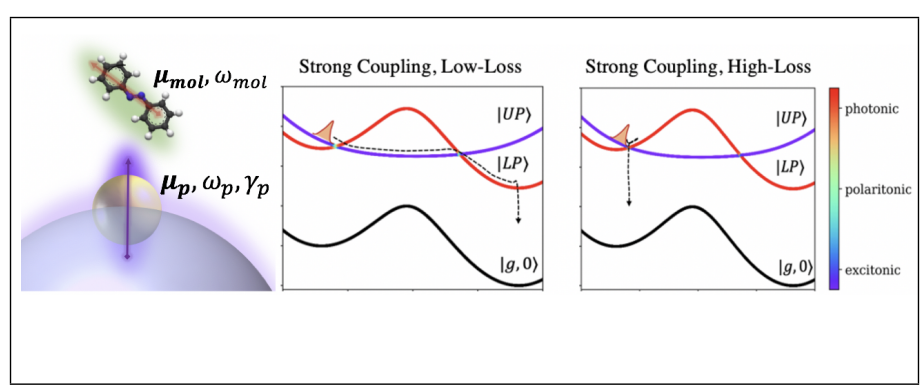

